\title{
Alarming levels of drug-resistant tuberculosis in Belarus: results of a
} survey in Minsk

\author{
Alena Skrahina*, Henadz Hurevich*, Aksana Zalutskaya*, Evgeni Sahalchyk*, \\ Andrei Astrauko*, Wayne van Gemert ${ }^{\#}$, Sven Hoffner", \\ Valiantsin Rusovich ${ }^{+}$and Matteo Zignol $^{\#}$
}

ABSTRACT: Resistance to anti-tuberculosis (TB) medicines is a major public health threat in most countries of the former Soviet Union. As no representative and quality-assured information on the magnitude of this problem existed in Belarus, a survey was conducted in the capital city of Minsk.

Between November 2009 and December 2010, 156 consecutively diagnosed new and 68 previously treated culture-positive TB patients residing in Minsk were enrolled in the survey. Mycobacterium tuberculosis isolates were obtained from each patient and tested for susceptibility to first- and second-line anti-TB drugs.

Multidrug-resistant (MDR)-TB was found in $35.3 \%(95 \% \mathrm{Cl} 27.7-42.8)$ of new patients and $76.5 \%$ (95\% Cl 66.1-86.8) of those previously treated. Overall, nearly one in two patients enrolled had MDR-TB. Extensively drug-resistant TB was reported in 15 of the 107 MDR-TB patients $(14.0 \%$, $95 \% \mathrm{Cl}$ 7.3-20.7). Patients $<35$ yrs of age have shown a two times higher odds ratio of multidrugresistant TB than those aged $>35$ yrs.

The findings of this survey in Minsk city are alarming and represent the highest proportions of MDR-TB ever recorded in the world. This study greatly contributes to the understanding of the burden of drug-resistant TB in urban areas of Belarus.

KEYWORDS: Epidemiology, extensively drug-resistant tuberculosis, multidrug-resistant tuberculosis, surveillance

$\mathbf{T}$ uberculosis (TB) in Belarus, as in most countries of the former Soviet Union, still represents an issue of serious public health concern [1-5]. Although over the past two decades the incidence rate of TB in the country has nearly halved, from 80 cases per 100,000 population in 1990 to 45 per 100,000 in 2010 [6], the emergence of forms of $\mathrm{TB}$ resistant to the most powerful medicines, including multidrugresistant (MDR)-TB (defined as TB caused by Mycobacterium tuberculosis resistant to isoniazid and rifampicin) [7] and extensively drug-resistant (XDR)-TB (defined as MDR-TB plus resistance to fluoroquinolones and at least one second-line injectable agent) [7], has been increasingly documented and may jeopardise the achievements made to date in controlling the community and nosocomial spread of $M$. tuberculosis strains. Official national statistics, while not sufficiently representative or quality assured, suggest that the

For editorial comments see page 1290. proportion of previously untreated (i.e. "new") TB cases having MDR-TB strains has increased from $8.8 \%$ in 2003 (169 cases) to $25.7 \%$ in 2010 (507 cases) [8-12].

Of the approximately 5,000 patients diagnosed with TB each year in Belarus, nearly 3,000 have a bacteriologically confirmed diagnosis, with the remaining 2,000 being diagnosed only on clinical and/or radiological evidence [6]. Patients with drug-sensitive TB are treated with the standard regimen proposed by the World Health Organization (WHO) since 2009 [13], with a treatment success rate for new smear-positive cases of $71 \%$ in that year, which is above the average of the WHO European Region [6]. A total of 1,576 patients with MDR-TB were started on treatment in 2010 in the country. The patients were treated in one of the 19 dedicated TB hospitals during the intensive phase of treatment (6-8 months) and
AFFILIATIONS

*Republican Scientific and Practical Centre for Pulmonology and Tuberculosis, and

+World Health Organization Country Office, Minsk, Belarus

\#Stop TB Dept, World Health Organization, Geneva, Switzerland. "Dept of Preparedness, Swedish Institute for Communicable Disease Control, Stockholm, Sweden.

CORRESPONDENCE

A. Skrahina

Republican Scientific and Practical Centre for Pulmonology and

Tuberculosis

Avenue Dolginovski trakt

157

220053 Minsk

Belarus

E-mail: alena_skrahina@tut.by

Received:

Aug 232011

Accepted after revision:

Sept 212011

First published online:

Oct 172011

This article was modified in April 2016 to correct errors in the licence information. 
then asked to continue their therapy in one of the 213 outpatient facilities for an additional 18-24 months, as per international recommendations [7, 13-15].

Very high frequencies of MDR-TB have been documented in nearby countries, including the Russian Federation [16], the Ukraine [17], Lithuania and Latvia [18]; reliable data from neighbouring Pskov Oblast in Russia found MDR-TB in 28\% of new TB cases in 2008 [19]. Given that Belarus was one of the few former Soviet Union Republics without sufficiently representative and quality-assured data on the magnitude of drug resistance, the Republican Scientific and Practical Centre for Pulmonology and Tuberculosis (Minsk, Belarus) decided to undertake a drug-resistance survey following WHO recommendations [20]. The capital city of Minsk, with a population of 1.8 million people, was selected as the target area for this study. The survey aimed to investigate levels and patterns of resistance to first- and second-line anti-TB drugs among new and previously treated TB cases and to explore risk factors for the development of drug resistance. Herein, we present the results of this survey.

\section{MATERIALS AND METHODS}

Between November 2009 and December 2010 a survey to investigate anti-TB drug resistance was implemented in the capital city of Minsk by the Republican Scientific and Practical Centre for Pulmonology and Tuberculosis and the National TB Reference Laboratory with technical support from WHO and the Swedish Institute for Communicable Disease Control (Stockholm, Sweden), which acts as the Supranational TB Reference Laboratory for Belarus [21]. The study was designed to generate representative information on drug resistance among all patients with TB in the capital city. All consecutive patients with newly diagnosed and previously treated culturepositive pulmonary TB residing in Minsk for $\geqslant 6$ months and registered in any of the three TB city dispensaries were invited to take part in the study. Only patients residing in Minsk city for $\geqslant 6$ months were considered eligible for enrolment to avoid the risk of including subjects living in other parts of the country who could have travelled to the capital city after diagnosis to seek better healthcare. New patients were defined as those without a history of prior treatment with first-line anti-TB drugs (isoniazid rifampicin, ethambutol and streptomycin), or those having received prior treatment for $<1$ month; previously treated TB cases were those who had received prior first-line anti-TB treatment for $\geqslant 1$ month [13, 20]. Informed consent was obtained from patients before enrolment into the study. The following variables were collected for each patient through a clinician-led interview performed during sputum collection: sex; age; country of birth; treatment history (new/previously treated, including subcategory of previous treatment); HIV status; education (university/college/secondary school/primary school or lower); size of household (number of persons living in the same house); history of imprisonment; and history of smoking in the past 5 yrs. Medical records were reviewed to confirm the reliability of the information on previous treatment history gathered during the interview. A random sample of patients (nearly $40 \%$ of the total) was re-interviewed during later monitoring visits to assess the quality of the answers provided in the initial interviews. Re-interviews showed full concordance with treatment history information obtained during the initial interviews.

Only patients with positive culture were included in the analysis. Patients with only clinical and/or radiological diagnosis of TB, extrapulmonary TB cases, cases of TB caused by mycobacteria other than $M$. tuberculosis, and patients having already received more than one re-treatment regimen were excluded. The ethics committee of the review board of the Republican Scientific and Practical Centre for Pulmonology and Tuberculosis reviewed and approved the survey protocol.

Two sputum specimens were collected from each patient. Isolation and identification were performed on BACTEC MGIT 960 (Becton, Dickinson and Company, Franklin Lakes, NJ, USA). Isolates of M. tuberculosis were sent to the National TB Reference Laboratory at the Republican Scientific and Practical Centre for Pulmonology and Tuberculosis for drug susceptibility testing using BACTEC MGIT 960 [22]. The following critical drug concentrations were used: isoniazid $0.1 \mu \mathrm{g} \cdot \mathrm{L}^{-1}$; rifampicin $1.0 \mu \mathrm{g} \cdot \mathrm{L}^{-1}$; ethambutol $5.0 \mu \mathrm{g} \cdot \mathrm{L}^{-1}$; streptomycin $1.0 \mu \mathrm{g} \cdot \mathrm{L}^{-1}$; kanamycin $2.5 \mu \mathrm{g} \cdot \mathrm{L}^{-1}$, amikacin $1.0 \mu \mathrm{g} \cdot \mathrm{L}^{-1}$; capreomycin $2.5 \mu \mathrm{g} \cdot \mathrm{L}^{-1}$; and ofloxacin $2.0 \mu \mathrm{g} \cdot \mathrm{L}^{-1}$.

The routine external quality assurance system for drugsusceptibility testing at the National TB Reference Laboratory includes annual proficiency testing and regular on-site monitoring missions by the partner Supranational TB Reference Laboratory. To further assure the quality of the survey results, a sample of susceptible and resistant strains was shipped to the Supranational TB Reference Laboratory in Stockholm for rechecking as per WHO recommendations [20]. In the proficiency testing exercise and in re-checking survey strains, concordance between the two laboratories was $100 \%$ for the most important first- and second-line drugs (isoniazid, rifampicin, kanamycin, amikacin and capreomycin).

Data were double-entered into EpiInfo (version 3.5.1; Centers for Disease Control and Prevention, Atlanta, GA, USA) and analysed using STATA statistical software (Release 10.0; Stata Corporation, College Station, TX, USA). Pearson's Chi-squared statistics or Fisher's two-tailed test were used to compare categorical variables. All variables were studied in a univariate analysis; those with $\mathrm{p}$-values $<0.05$ were included in a multivariate logistic regression analysis. Confounding effects were checked using backward regression analysis (a cut off of $\mathrm{p}<0.05$ was used to exclude variables from the model). In all analyses, confidence intervals and p-values were corrected for finite population and clustering effect.

\section{RESULTS}

During the intake period, a total of 465 patients were notified in the city of Minsk. Of them 235 (50.5\%) met the inclusion criteria of the survey (culture-positive pulmonary TB). Drug susceptibility tests could be performed for 224 (95.3\%) of these patients. Nine $(3.8 \%)$ patients had to be excluded because of culture contamination and two $(0.9 \%)$ because of growth of mycobacteria other than $M$. tuberculosis (fig. 1). Of the remaining 224 patients, 156 (69.6\%) were new TB cases and 68 (30.4\%) were patients with a history of previous TB treatment; 157 (70.1\%) were male. The median (range) age was 43 (19-87) yrs. Data on history of TB treatment and additional characteristics of the study population are shown in table 1. 


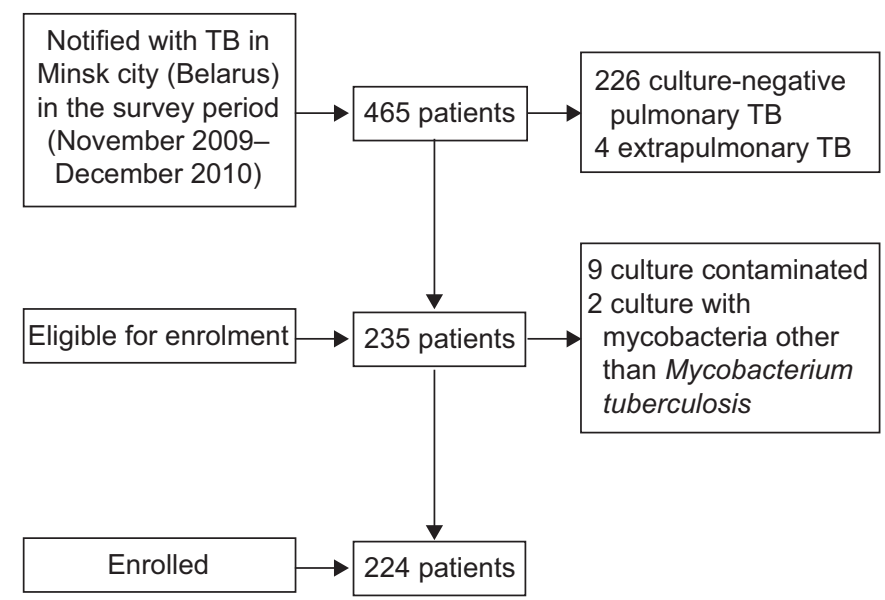

FIGURE 1. Selection of the study population. TB: tuberculosis.

Of the 156 isolates from new TB cases, 87 (55.8\%, 95\% CI 47.963.6) were resistant to at least one first-line drug and $55(35.3 \%$, 95\% CI 27.7-42.8) were found to have MDR-TB. Resistance to isoniazid, rifampicin, ethambutol and streptomycin in new cases is shown in table 2. Among those with MDR-TB, 5.5\% (95\% CI 0.0-11.7) had XDR-TB strains. Resistance to ofloxacin, kanamycin, amikacin and capreomycin in these TB and MDRTB cases is shown in table 3 .

Of the 68 isolates from previously treated TB cases, resistance to any first-line anti-TB drug was found in 56 cases $(82.4 \%, 95 \%$ CI 73.1-91.6) and MDR-TB in 52 cases (76.5\%, 95\% CI 66.186.8). Resistance to isoniazid, rifampicin, ethambutol and streptomycin in previously treated cases is shown in table 2.

Among those with MDR-TB, 23.1\% (95\% CI 11.2-34.9) had XDR-TB strains. Resistance to ofloxacin, kanamycin, amikacin and capreomycin in these previously treated TB and MDR-TB cases is shown in table 3 .

\begin{tabular}{lcccc} 
TABLE 1 & Characteristics of tuberculosis patients & \\
& Male & Female & Total & p-value \\
\hline & & & & \\
Total subjects & $157(70.1)$ & $67(29.9)$ & $224(100.0)$ & \\
Age group yrs & & & & 0.000 \\
$\quad \leqslant 24$ & $7(4.5)$ & $13(19.4)$ & $20(8.9)$ & \\
$25-34$ & $32(20.4)$ & $22(32.8)$ & $54(24.1)$ & \\
$35-44$ & $39(24.8)$ & $13(19.4)$ & $52(23.2)$ & \\
$45-54$ & $52(33.1)$ & $9(13.4)$ & $61(27.2)$ & \\
$55-64$ & $20(12.7)$ & $2(3.0)$ & $22(9.8)$ & \\
$\geqslant 65$ & $7(4.5)$ & $8(11.9)$ & $15(6.7)$ & \\
Country of birth & & & & 0.632 \\
Belarus & $150(95.5)$ & $63(94.0)$ & $213(95.1)$ & \\
Abroad & $7(4.5)$ & $4(6.0)$ & $11(4.9)$ & \\
Treatment history & & & & 0.458 \\
$\quad$ New & $107(68.2)$ & $49(73.1)$ & $156(69.6)$ & \\
Previously treated & $50(31.9)$ & $18(26.9)$ & $68(30.4)$ & \\
& & & & \\
& & &
\end{tabular}

Data are presented as $n(\%)$, unless otherwise stated.
For 15 isolates (nine from new and six from previously treated TB cases) second-line drug susceptibility testing was not available due to test failure of BACTEC MGIT 960. Tests were not repeated as those isolates were not MDR-TB.

As expected, previously treated TB cases in whom treatment with second-line drugs had failed showed a significantly higher risk of MDR-TB compared to relapse cases, i.e. patients that come back with TB after having been previously declared cured or having successfully completed treatment (OR 10.6, 95\% CI 5.1-22.3; $\mathrm{p}<0.001)$. Interestingly, default cases, i.e. patients who come back with TB after having interrupted treatment, also had a significantly higher risk of MDR-TB compared to relapse cases (OR 2.9, 95\% CI 1.8-4.8; p<0.001). Compared to new TB cases, however, relapse cases were found to have a significantly higher proportion of MDR-TB (OR 2.9, $95 \%$ CI $1.2-7.7 ; p=0.011$ ), with $61.5 \%$ (95\% CI $41.5-81.6)$ versus $35.3 \%$ (95\% CI $27.7-42.8)$ in new cases.

Overall, among the 224 patients enrolled in the study, MDR-TB was found in $47.8 \%$ (95\% CI 41.2-54.4) (table 2). Among these $14.0 \%$ (95\% CI 7.3-20.7) were found to have XDR-TB (table 3).

Although three TB patients with HIV infection were registered during the study period, none were enrolled in the survey as their sputum specimens were culture negative.

As expected, history of previous anti-TB treatment was the strongest independent factor for MDR-TB (OR 7.4, 95\% CI 4.3$12.7 ; \mathrm{p}<0.001)$. Among new TB cases, the risk of MDR-TB in the $\leqslant 24$ to 34 -yr age group was two times higher than in the $\geqslant 35$-yr age group (OR 2.0, 95\% CI 1.3-3.1; $\mathrm{p}=0.002$ ). Associations between MDR-TB and sex, country of birth, education, size of the household, history of imprisonment and smoking in the past 5 yrs were not found to be statistically significant (table 4).

\section{DISCUSSION}

This study has revealed extremely high levels of drug-resistant $\mathrm{TB}$ in the city of Minsk. The proportions of new and previously treated TB cases found to have MDR-TB (35.3\% and $76.5 \%$, respectively) are the highest recorded anywhere in the world in the history of the WHO Global Project on Anti-tuberculosis Drug Resistance Surveillance [19, 23]. The study has also revealed worrisome proportions of XDR-TB among new TB

\begin{tabular}{|c|c|c|c|}
\hline TABLE 2 & $\begin{array}{l}\text { Resistance to fi } \\
\text { previously treat }\end{array}$ & $\begin{array}{l}\text { st-line drugs amon } \\
\text { d and all tubercula }\end{array}$ & $\begin{array}{l}\text { new, } \\
\text { is cases }\end{array}$ \\
\hline Resistance & $\mathrm{New}^{\#}$ & Previously treated" & $\mathrm{All}^{+}$ \\
\hline Any & $55.8(47.9-63.6)$ & $82.4(73.1-91.6)$ & $63.8(57.5-70.2)$ \\
\hline $\mathbf{H}$ & $47.4(39.5-55.4)$ & $79.4(69.6-89.3)$ & $57.1(50.6-63.7)$ \\
\hline $\mathbf{R}$ & 36.5 (28.9-44.2) & $77.9(67.8-88.1)$ & $49.1(42.5-55.7)$ \\
\hline MDR & $35.3(27.7-42.8)$ & $76.5(66.1-86.8)$ & $47.8(41.2-54.4)$ \\
\hline $\mathbf{E}$ & $23.1(16.4-29.8)$ & $52.9(40.8-65.1)$ & $32.1(26.0-38.3)$ \\
\hline $\mathbf{S}$ & $53.2(45.3-61.1)$ & 77.9 (67.8-88.1) & $60.7(54.3-67.2)$ \\
\hline
\end{tabular}




\begin{tabular}{|c|c|c|c|c|c|c|}
\hline TABLE 3 & $\begin{array}{l}\text { ce to second } \\
\text { nd among ne }\end{array}$ & $\begin{array}{l}\text { e drugs among ne } \\
\text { previously treated }\end{array}$ & $\begin{array}{l}\text { previously trea } \\
d \text { all TB cases }\end{array}$ & and all multi & g-resistant (MDR) & perculosis (TB) \\
\hline \multirow[t]{2}{*}{ Resistance } & \multicolumn{3}{|c|}{ Cases with MDR-TB } & \multicolumn{3}{|c|}{ All cases with $\mathrm{TB}^{\S}$} \\
\hline & New* & Previously treated & $\mathrm{All}^{+}$ & New & Previously treated & All \\
\hline Ofx & $14.5(4.9-24.2)$ & $46.2(32.1-60.2)$ & $29.9(21.1-38.7)$ & $6.1(2.2-10.0)$ & $38.7(26.2-51.2)$ & $15.8(10.8-20.8)$ \\
\hline Amk & $16.4(6.3-26.5)$ & $26.9(14.5-39.4)$ & $21.5(13.6-29.4)$ & $6.8(2.7-10.9)$ & $24.2(13.2-35.2)$ & $12.0(7.5-16.4)$ \\
\hline $\mathrm{Cm}$ & $16.4(6.3-26.5)$ & $25.0(12.8-37.2)$ & $20.6(12.8-28.3)$ & $6.9(2.7-11.1)$ & $21.3(10.7-31.9)$ & $11.2(6.9-15.6)$ \\
\hline Injectable agents ${ }^{f}$ & $18.2(7.7-28.7)$ & $36.5(23.0-50.1)$ & $27.1(18.5-35.7)$ & $7.5(3.2-11.8)$ & $32.3(20.3-44.2)$ & $14.8(10.0-19.7)$ \\
\hline XDR & $5.5(0.0-11.7)$ & $23.1(11.2-34.9)$ & $14.0(7.3-20.7)$ & $2.0(0.0-4.4)$ & $19.4(9.2-29.5)$ & $7.2(3.6-10.7)$ \\
\hline
\end{tabular}

cases $(2.0 \%)$ and in previously treated cases (19.4\%): proportions more common for MDR-TB in other parts of the world $[19,23]$.

The very high frequency of drug-resistant forms of TB indicates the existence of serious problems in the organisation of TB treatment in Minsk, as well as in Belarus overall. The turbulent years following the dissolution of the Soviet Union were characterised by drug shortages and previously unseen social problems and poverty. As a result, a significant pool of drug-resistant strains emerged that continue to limit the success of the National TB control programme today, as we are still being confronted by several challenges.

First, the outpatient phase of TB treatment, and particularly MDR-TB treatment, is not managed strongly enough: in some locations where patients receive ambulatory treatment, costly second-line drugs are insufficient and supplied irregularly. Ambulatory patients on any treatment regimen, first or second line, frequently default due to the inconvenience or cost of travelling to obtain drugs and having to miss work, as well as coexisting social problems, including untreated alcohol addiction, which is common among TB patients in this region of the world.

Secondly, diagnosis of drug resistance is often a timely process, as solid culture and drug-susceptibility testing methods are most commonly used in the country. More rapid diagnostic methods, such as liquid culture and line probe assays [24], are not yet widely implemented, and Xpert MTB/RIF (Cepheid, Sunnydale, CA, USA) [25] has not yet been introduced. Implementation of rapid diagnostics, while more expensive than solid culture and conventional drug-susceptibility testing, could provide critical information to clinicians faster, allowing the patient to start appropriate treatment regimens sooner and prevent acquisition of further resistance and limit transmission of resistant $M$. tuberculosis strains.

Thirdly, infection control measures in TB hospitals and dispensaries, including administrative and individual protection measures, are frequently insufficient, resulting in transmission of strains from drug-resistant to drug-susceptible patients.
The magnitude of the problem of drug-resistant TB does not come as a complete surprise for the country, given its relatively advanced nationwide surveillance system that routinely tests for drug susceptibility in 22 laboratories (three in the city of Minsk) and centrally collects data at the Republican Scientific and Practical Centre for Pulmonology and Tuberculosis. This system has shown increasingly high proportions of drug resistance in recent years (e.g. 26.9\% of MDR-TB among new cases in Minsk in 2009) [8-11]. However, the study described herein has ensured high-quality testing, centrally performed at the National TB Reference Laboratory and confirmed by the partner Supranational TB Reference Laboratory, and careful collection of accurate patient treatment history information.

The routine surveillance system reported considerably lower proportions of MDR-TB than those found in this study: $26.9 \%$ versus $35.3 \%$ among new TB cases and $60.3 \%$ versus $76.1 \%$ among previously treated cases [8-11]. The differences may be due to shortcomings in registration of patient treatment histories in the routine surveillance system, or potential weaknesses in laboratory performance of the other two laboratories that routinely perform drug susceptibility testing in Minsk. The insights gained from the study will strengthen the routine surveillance system.

When disaggregating previously treated TB cases by subcategory, frequencies of MDR-TB were found to be significantly higher among relapse cases than new TB cases, $61.5 \%$ versus $35.3 \%$, respectively. This difference reflects that weakness may exist in procedures for declaring patients successfully treated, i.e. such patients were not successfully treated for TB in the first place due to remaining drug-resistant bacteria, and/or that inadequate infection control measures may be commonly causing TB patients to be re-infected with their fellow patients' drug-resistant strains during hospitalisation.

A single data point in time, as has been provided in this survey, cannot answer the question of whether the situation of drug-resistant TB is getting better or worse in the city of Minsk. However, the higher prevalence of MDR-TB among the younger age groups of previously untreated TB cases is an indication of a possible increasing transmission of MDR-TB strains, as previously untreated TB cases from older age 
TABLE 4 Risk factors for multidrug-resistant tuberculosis (MDR-TB)

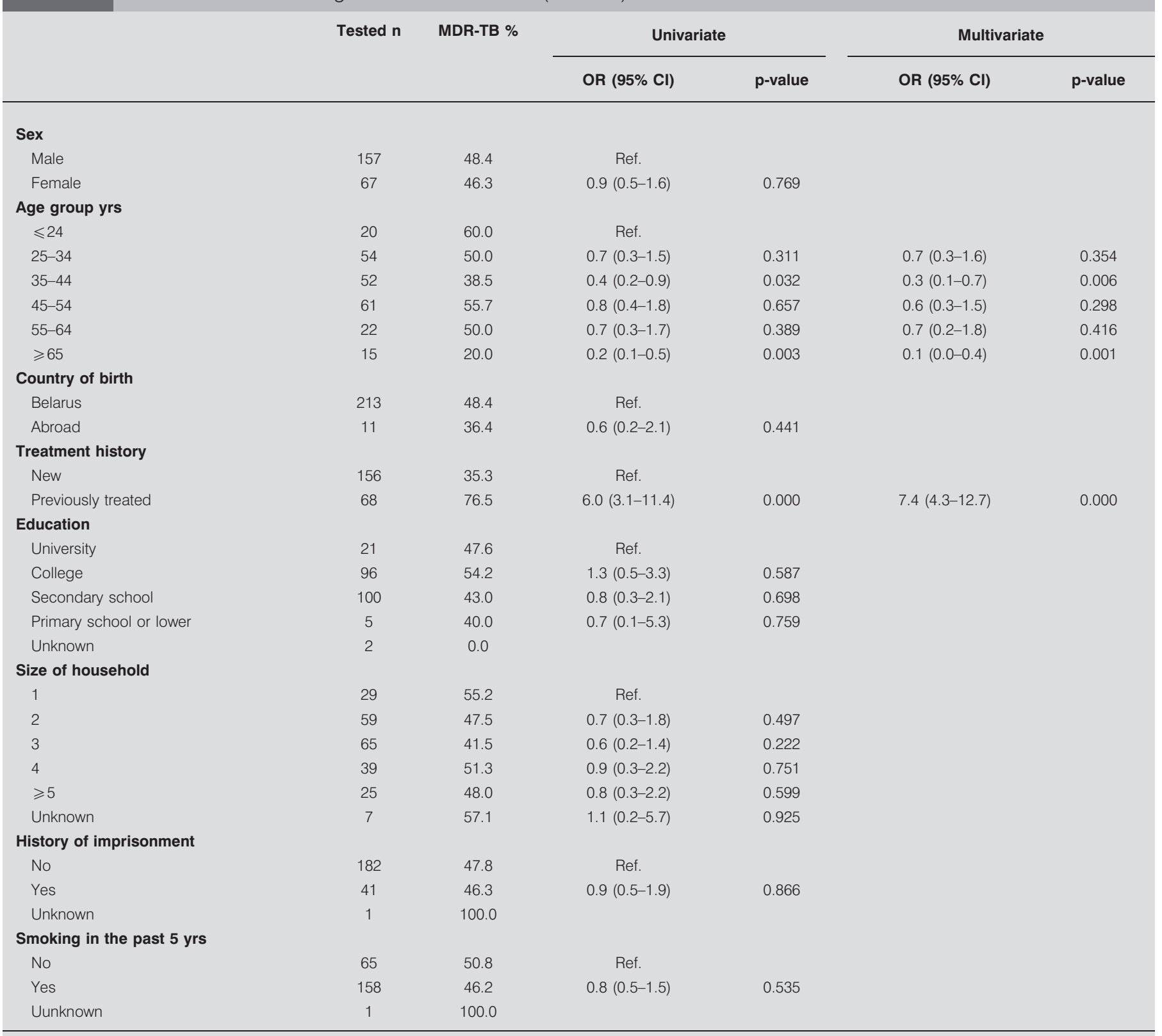

Ref.: reference.

groups (found to have lower proportions of MDR-TB) may have been infected with strains decades ago. This hypothesis is supported by national routine surveillance data that in recent years have shown a worsening situation of MDR-TB, with an increasing absolute number of MDR-TB cases. At the same time, the overall incidence rate of $\mathrm{TB}$ in the country has dropped [6], implying that the TB control programme is having considerable success at treating drug-susceptible TB, but is challenged by a growing pool of drug-resistant patients who represent a reservoir of infection to others. The sharp increase in proportion of MDR-TB among new TB cases in Belarus reported by the national surveillance system (form $8.8 \%$ in 2003 to $25.7 \%$ in 2010) can be attributed to a real growth in numbers of drug-resistant cases but also to the decrease in the number of drug-susceptible TB cases and to the improvement in laboratory services in recent years that has resulted in greater access to diagnosis.

An important limitation of this study is that patients who had failed a standardised re-treatment regimen for $\mathrm{TB}$ and were subsequently on other non-standardised regimens were excluded from the study. This was performed in line with WHO recommendations to avoid including patients who had already been started on treatment [20]. In most countries those cases are few but in Belarus, as in many other former Soviet countries, such cases (commonly called "chronic" patients) are 
common and generally have a high frequency of drug resistance. Disregarding such cases that contribute to the overall burden of drug resistance results in underestimating the gravity of the problem in Minsk.

Significant gaps exist in the geographic coverage of reliable antiTB drug-resistance surveillance in the former Soviet Union, including Kyrgyzstan, most of the Ukraine, Uzbekistan, Tajikistan and Turkmenistan, and large swaths of Russia. It is therefore entirely possible that other settings in the former Soviet Union unknowingly have similarly high or even higher proportions of drug-resistant TB than what has been reported in Minsk. Additionally, a number of settings only have old data, and many countries with nationwide data lack precise data at the provincial or city level $[19,23]$.

At the time of publication of this report, Belarus is undertaking a nationwide survey of drug resistance, knowing the value of the information gained from the Minsk survey and taking advantage of the experience gained. It is imperative that all countries recognise the importance of drug-resistance surveillance as an integral component of TB control [26, 27]. Measures need to be taken to strengthen laboratories and recording and reporting systems, and to organise special surveys as needed, in order to establish drug-resistance surveillance systems that provide representative and accurate data for informed decision making.

\section{SUPPORT STATEMENT}

This study was supported by the US Agency for International Development through a grant to the World Health Organization.

\section{STATEMENT OF INTEREST}

None declared.

\section{REFERENCES}

1 Kliiman K, Altraja A. Predictors of poor treatment outcome in multi- and extensively drug-resistant pulmonary TB. Eur Respir J 2009; 33: 1085-1094.

2 Leimane V, Dravniece G, Riekstina V, et al. Treatment outcome of multidrug/extensively drug-resistant tuberculosis in Latvia, 20002004. Eur Respir J 2010; 36: 584-593.

3 Migliori GB, Sotgiu G, Lange C, et al. Extensively drug-resistant tuberculosis: back to the future. Eur Respir J 2010; 36: 475477.

4 Migliori GB, Besozzi G, Girardi E, et al. Clinical and operational value of the extensively drug-resistant tuberculosis definition. Eur Respir J 2007; 30: 623-626.

5 Sotgiu G, Ferrara G, Matteelli A, et al. Epidemiology and clinical management of XDR-TB: a systematic review by TBNET. Eur Respir J 2009; 33: 871-881.

6 World Health Organization. Global tuberculosis control: WHO report 2010. WHO/HTM/TB/2010.7. Geneva, World Health Organization. Available from: http://whqlibdoc.who.int/publications /2010/9789241564069_eng.pdf

7 World Health Organization. Guidelines for the programmatic management of drug-resistant tuberculosis: emergency update. WHO/HTM/TB/2008.402. Geneva, World Health Organization. Available from: http://whqlibdoc.who.int/publications/2008/ 9789241547581_eng.pdf

8 Skrahina A, Astrauko A, Hurevich H, et al. Multidrug resistance and its influence on TB treatment results in the Republic of Belarus. Lechebnoe Delo (Minsk). Issue 5 (15), 2010; pp. 17-21.
9 Skrahina A, Astrauko A, Hurevich H. Tuberculosis and drug resistant tuberculosis monitoring system. Proceedings of the 4th International Research and Practical Conference on Actual Problems of Penitentiary Medicine, Tuberculosis and Other Socially Important Diseases in Prisons (Minsk, September 2009); pp. 88-93.

10 Skrahina A, Astrauko A, Hurevich H. Drug resistance trends among tuberculosis patients registered in dispensaries. Proceedings of the 4th International Research and Practical Conference on Actual Problems of Penitentiary Medicine, Tuberculosis and Other Socially Important Diseases in Prisons (Minsk, September 2009); pp. 75-81.

11 Skrahina A, Astrauko A, Hurevich $\mathrm{H}$. The problem of multidrug resistance among $\mathrm{TB}$ and $\mathrm{TB} / \mathrm{HIV}$ patients in the Republic of Belarus. Proceedings of the 7th Congress of Physiologists of Belarus TB diagnostic and treatment in light of DOTS strategy (Minsk, May 2008); pp. 69-90.

12 Public Health Ministry of the Republic of Belarus. Public Health in the Republic of Belarus: An Official Statistics Collection 2010 Available from: http://med.by/content/stat/stat2011/2010_1. pdf

13 World Health Organization. Treatment of tuberculosis: guidelines for national programmes. 4th Edn. WHO/HTM/TB/ 2009.420. Geneva, World Health Organization, 2009. Available from: http://whqlibdoc.who.int/publications/2010/9789241547 833_eng.pdf

14 World Health Organization. Guidelines for the programmatic management of drug-resistant tuberculosis: 2011 update. WHO/ HTM/TB/2011.6. Geneva, World Health Organization. Available from: http://whqlibdoc.who.int/publications/2011/97892415015 83_eng.pdf

15 Falzon D, Jaramillo E, Schünemann $\mathrm{H}$, et al. WHO guidelines for the programmatic management of drug-resistant tuberculosis: 2011 update. Eur Respir J 2011; 38: 516-528.

16 Ministry of Health of the Russian Federation. Tuberculosis in the Russian Federation, 2008. An analytical review of the TB statistical indicators used in the Russian Federation. Moscow, Ministry of Health of the Russian Federation, 2009.

17 Dubrovina I, Miskinis K, Lyepshina S, et al. Drug-resistant tuberculosis and HIV in Ukraine: a threatening convergence of two epidemics? Int J Tuberc Lung Dis 2008; 12: 756-762.

18 World Health Organization. Towards universal access to diagnosis and treatment of multidrug-resistant and extensively drugresistant tuberculosis by 2015. WHO/HTM/TB/2011.3. Geneva, World Health Organization. Available from: http://whqlibdoc. who.int/publications/2011/9789241501330_eng.pdf

19 World Health Organization. Multidrug and extensively drugresistant TB (M/XDR-TB): 2010 Global Report on Surveillance and Response. WHO/HTM/TB/2010.3. Geneva, World Health Organization. Available from: http://whqlibdoc.who.int/publications/ 2010/9789241599191_eng.pdf

20 World Health Organization. Guidelines for surveillance of drug resistance in tuberculosis. 4th Edn. WHO/HTM/TB/2009.422. Geneva, World Health Organization. Available from: http:// whqlibdoc.who.int/publications/2009/9789241598675_eng.pdf

21 Van Deun A, Wright A, Zignol M, et al. Drug susceptibility testing proficiency in the network of supranational tuberculosis reference laboratories. Int J Tuberc Lung Dis 2011; 15: 116-124.

22 Krüüner A, Yates MD, Drobniewski FA. Evaluation of MGIT 960-based antimicrobial testing and determination of critical concentrations of first- and second-line antimicrobial drugs with drug-resistant clinical strains of Mycobacterium tuberculosis. J Clin Microbiol 2006; 44: 811-818.

23 Wright A, Zignol M, Van Deun A, et al. Epidemiology of antituberculosis drug resistance 2002-07: an updated analysis of the 
Global Project on Anti-Tuberculosis Drug Resistance Surveillance. Lancet 2009; 373: 1861-1873.

24 Skenders G, Fry AM, Prokopovica I, et al. Multidrug-resistant tuberculosis detection, Latvia. Emerg Infect Dis 2005; 11: 1461-1463.

25 Boehme CC, Nabeta P, Hillemann D, et al. Rapid molecular detection of tuberculosis and rifampin resistance. $N$ Engl J Med 2010; 363: 1005-1015.
26 Zignol M, van Gemert W, Falzon D, et al. Modernizing surveillance of anti-tuberculosis drug resistance: from special surveys to routine testing. Clin Infect Dis 2011; 52: 901-906.

27 Stop TB Partnership, World Health Organization. The Global Plan to Stop TB 2011-2015. Transforming the fight towards elimination of tuberculosis. Geneva, World Health Organization, 2010. Available from: www.stoptb.org/assets/documents/global/plan/ TB_GlobalPlanToStopTB2011-2015.pdf 